\title{
Error Estimates \\ for Semidiscrete Finite Element Methods for Parabolic Integro-Differential Equations
}

\author{
By Vidar Thomée and Nai-Ying Zhang
}

\begin{abstract}
The purpose of this paper is to attempt to carry over known results for spatially discrete finite element methods for linear parabolic equations to integro-differential equations of parabolic type with an integral kernel consisting of a partial differential operator of order $\beta \leq 2$. It is shown first that this is possible without restrictions when the exact solution is smooth. In the case of a homogeneous equation with nonsmooth initial data $v, v \in L_{2}$, optimal $O\left(h^{r}\right)$ convergence for positive time is possible in general only if $r \leq 4-\beta$. This depends on the fact that the exact solution is then only in $H^{4-\beta}$.
\end{abstract}

1. Introduction. The aim of this paper is to analyze spatially discrete finite element methods for solving initial-boundary value problems of the form

$$
\begin{aligned}
& u_{t}+A u=\int_{0}^{t} B(t, s) u(s) d s+f \equiv \tilde{B} u+f \text { in } \Omega \times J, \\
& u=0 \text { on } \partial \Omega \times J \\
& u(\cdot, 0)=v \text { in } \Omega .
\end{aligned}
$$

Here, $u=u(x, t)$ is a function in $\bar{\Omega} \times \bar{J}$, where $\Omega$ is a bounded domain in $R^{d}$ with a smooth boundary $\partial \Omega, J=(0, \bar{t}]$ with $\bar{t}>0$, and $u_{t}=\frac{\partial u}{\partial t}$. Further, $A$ is a second-order elliptic partial differential operator,

$$
A=-\sum_{i, j=1}^{d} \frac{\partial}{\partial x_{j}}\left(a_{i j}(x) \frac{\partial}{\partial x_{i}}\right)+a_{0}(x) I,
$$

where $\left(a_{i j}\right)$ is a time-independent matrix, which is symmetric and uniformly positive definite in $\bar{\Omega}, a_{0}(x) \geq 0$ in $\bar{\Omega}$, and $B=B(t, s)$ is a general second-order partial differential operator of order $\beta \leq 2$,

$$
B(t, s)=-\sum_{i, j=1}^{d} \frac{\partial}{\partial x_{j}}\left(b_{i j}(x ; t, s) \frac{\partial}{\partial x_{i}}\right)+\sum_{j=1}^{d} b_{j}(x ; t, s) \frac{\partial}{\partial x_{j}}+b_{0}(x ; t, s) I,
$$

and $\tilde{B} u=\tilde{B} u(t)$ stands for the integral term in (1.1). Finally, $f$ and $v$ are prescribed real-valued functions. Throughout this paper, we shall assume that $f$ and the coefficients of $A$ and $B$ are smooth.

Parabolic integro-differential equations (PIDE) of the above type, and nonlinear variants thereof, arise in many applications, such as, for instance, in non-Fourier

Received May 9, 1988.

1980 Mathematics Subject Classification (1985 Revision). Primary 65M60, 65R20.

Key words and phrases. Integro-differential equation, parabolic, nonsmooth data, regularity estimates, finite elements, Galerkin, semidiscrete, error estimates. 
models for heat conduction in materials with memory and in the theory of nuclear reactors; see, e.g., the introduction in Greenwell Yanik and Fairweather [3], where also references to studies of existence, uniqueness and regularity are given.

For the purpose of numerical solution we assume that we are given a family $\left\{S_{h}\right\}$ of finite-dimensional subspaces of $H_{0}^{1}=H_{0}^{1}(\Omega)$ such that, with $r$ a given integer $\geq 2$,

$$
\inf _{\chi \in S_{h}}\left\{\|u-\chi\|+h\|u-\chi\|_{1}\right\} \leq C h^{s}\|u\|_{s} \quad \text { for } 1 \leq s \leq r, \text { if } u \in H^{s} \cap H_{0}^{1} .
$$

Here and below we work in the standard Sobolev spaces $H^{s}=H^{s}(\Omega)$, the norms in which are denoted $\|\cdot\|_{s}$, with $s$ omitted when zero so that $\|\cdot\|$ is the norm in $L_{2}=L_{2}(\Omega)$. No inverse assumption is used for $\left\{S_{h}\right\}$.

The semidiscrete Galerkin finite element method that we shall study is then to find $u_{h}: \bar{J} \rightarrow S_{h}$ such that

$$
\begin{aligned}
\left(u_{h, t}, \chi\right)+A\left(u_{h}, \chi\right) & =\int_{0}^{t} B\left(t, s ; u_{h}(s), \chi\right) d s+(f, \chi) \\
& \equiv \tilde{B}\left(u_{h}, \chi\right)+(f, \chi) \quad \forall \chi \in S_{h}, t \in J \\
u_{h}(0)=v_{h} . &
\end{aligned}
$$

Here, $v_{h}$ is an appropriate approximation of $v$ in $S_{h},(\cdot, \cdot)$ is the standard inner product in $L_{2}, A(\cdot, \cdot)$ and $B(t, s ; \cdot, \cdot)$ are the bilinear forms associated with the operators $A$ and $B(t, s)$, i.e.,

$$
A(u, w)=\int_{\Omega}\left(\sum_{i, j=1}^{d} a_{i j}(x) \frac{\partial u}{\partial x_{i}} \frac{\partial w}{\partial x_{j}}+a_{0} u w\right) d x
$$

and

$$
\begin{aligned}
& B(t, s ; u, w) \\
& \quad=\int_{\Omega}\left(\sum_{i, j=1}^{d} b_{i j}(x ; t, s) \frac{\partial u}{\partial x_{i}} \frac{\partial w}{\partial x_{j}}+\sum_{j=1}^{d} b_{j}(x ; t, s) \frac{\partial u}{\partial x_{j}} w+b_{0}(x ; t, s) u w\right) d x
\end{aligned}
$$

and $\tilde{B}(\cdot, \cdot)=\tilde{B}(t ; \cdot, \cdot)$ is defined by $(1.3)$.

Our purpose here is to discuss to what extent known error estimates for the case of a parabolic differential equation (cf., e.g., Thomée [7]) carry over to the present situation.

We shall consider first, in Section 2 below, the case of a smooth solution, i.e., when the smoothness of the exact solution is sufficient not to cause any complications in the analysis. We shall then be able to show that the result for $B=0$ remains valid, i.e., that

$$
\left\|u_{h}(t)-u(t)\right\| \leq C\left\|v_{h}-v\right\|+C h^{r}\left\{\|v\|_{r}+\int_{0}^{t}\left\|u_{t}\right\|_{r} d s\right\} \quad \text { for } t \in \bar{J} .
$$

We shall then turn to the homogeneous equation $(f=0)$ with nonsmooth initial data. For the differential equation case, it is known that if $v_{h}$ is chosen as $P_{h} v$, the $L_{2}$-projection of $v$ onto $S_{h}$, then

$$
\left\|u_{h}(t)-u(t)\right\| \leq C h^{r} t^{-r / 2}\|v\| \quad \text { for } t \in J
$$


thus showing optimal-order convergence for positive $t$, even with $v$ only in $L_{2}$. This is related to the fact that the solution of a homogeneous parabolic equation is smooth for positive $t$, even when the initial data are not. In quantitative form, this may be expressed by the inequality

$$
\|u(t)\|_{\alpha} \leq C t^{-\alpha / 2}\|v\| \quad \text { for } t \in J
$$

which is valid for any $\alpha \geq 0$.

Therefore, the first point on the agenda is to investigate the smoothness of the solution in the case $f=0$ of $(1.1)$, when $v$ is nonsmooth. It turns out that in the PIDE case the inequality (1.5) remains valid, but in general only for $\alpha \leq 4-\beta$, where $\beta$ is the order of $B(t, s)$. This is shown in Section 3 below.

It is natural that this smoothness property will be significant also in the study of the error in the semidiscrete solution. Our result is now that the error estimate (1.4) remains valid if $r \leq 4-\beta$, or, more precisely, with $r$ in (1.4) replaced by $\gamma=\min (4-\beta, r)$. This will be shown in Section 4 .

Earlier related results have been presented by Greenwell Yanik and Fairweather [3], who derived optimal-order error estimates in the case of a (nonlinear) problem with smooth solution, and with $\beta \leq 1$. An alternative proof of our smooth data result with $\beta=2$ has been given recently by Cannon and Lin [2]. Both smooth and nonsmooth data estimates have been demonstrated in Le Roux and Thomée [4] for a semilinear problem with $\beta=0$. For time stepping with special emphasis on economical quadrature, see Sloan and Thomée [5] (and also [4]).

We shall end this introduction by fixing our notation and collecting some material concerning the differential equation case of (1.1), i.e., the case $B=0$. In addition to $H^{r}$, we shall use the space $\dot{H}^{s}=\dot{H}^{s}(\Omega), s \geq 0$, defined by the norm $|v|_{s}=\left\|A^{s / 2} v\right\|$. We recall that for $s$ an integer, $\dot{H}^{s}=\left\{v \in H^{s} ; A^{j} v=0\right.$ on $\partial \Omega$ for $\left.j<s / 2\right\}$, and that the norms $\|\cdot\|_{s}$ and $|\cdot|_{s}$ are equivalent on $\dot{H}^{s}$ (cf. [7]).

Let thus $E(t)$ denote the semigroup on $L_{2}$ generated by the elliptic operator $A$, under homogeneous Dirichlet boundary conditions. The solution of the homogeneous parabolic equation with initial data $v$ is then $u(t)=E(t) v$ and has the property

$$
\left|\left(\frac{d}{d t}\right)^{j} E(t) v\right|_{q} \leq C t^{-(q-p) / 2-j}|v|_{p} \text { for } v \in \dot{H}^{p}, t \in J, 0 \leq p \leq q, j \geq 0 .
$$

Let further $E_{h}(t)$ denote the finite element analogue of $E(t)$, thus defined by the semidiscrete equation (1.3) with $f=0, B=0$. This operator on $S_{h}$ may be defined alternatively as the semigroup generated by the discrete analogue $A_{h}: S_{h} \rightarrow S_{h}$ of $A$, where

$$
\left(A_{h} \psi, \chi\right)=A(\psi, \chi) \quad \forall \psi, \chi \in S_{h}
$$

The error in the semidiscrete solution is thus $u_{h}(t)-u(t)=E_{h}(t) v_{h}-E(t) v$. In the particular case that $v_{h}=P_{h} v$, the $L_{2}$-projection of $v$ onto $S_{h}$, we shall use the error operator $F_{h}(t)=E_{h}(t) P_{h}-E(t)$. For this operator it is known that (cf. Theorem 3.1 of [1])

$$
\left\|F_{h}(t) v\right\| \leq C h^{s} t^{-(s-p) / 2}|v|_{p}, \quad 0 \leq p \leq s \leq r .
$$


Here and below, when $q>0$, we write $\|v\|_{-q}$ and $|v|_{-q}$ for the dual norms to $\|v\|_{q}$ and $|v|_{q}$ with respect to the $L_{2}$ inner product.

Related to the definition of the discrete elliptic operator $A_{h}$ is that of the solution operator $T_{h}: L_{2} \rightarrow S_{h}$ of the discrete elliptic problem, namely

$$
A\left(T_{h} f, \chi\right)=(f, \chi) \quad \forall \chi \in S_{h}
$$

it approximates the exact solution operator $T=A^{-1}: L_{2} \rightarrow \dot{H}^{2}$ in the sense that (1.8) $\left\|T_{h} f-T f\right\|_{-q} \leq C h^{p+q+2}\|f\|_{p}$ for $f \in H^{p}, 0 \leq p \leq r-2,-1 \leq q \leq r-2$.

The operator $T$ is selfadjoint and positive definite on $L_{2}$, and $T_{h}$ is selfadjoint, positive semidefinite on $L_{2}$ and positive definite on $S_{h}$. We also recall the elliptic regularity property $T: H^{q} \rightarrow H^{q+2} \cap H_{0}^{1}$ and the associated inequality

$$
\|T f\|_{q+2} \leq C\|f\|_{q} \text { for } f \in H^{q}, q \geq 0 .
$$

We finally recall the Ritz projection $R_{h}: H_{0}^{1} \rightarrow S_{h}$ defined by

$$
A\left(R_{h} u, \chi\right)=A(u, \chi) \quad \forall \chi \in S_{h} .
$$

In the appropriate domain we have $R_{h} v=T_{h} A v$, and, by (1.9), the inequality (1.8) may also be expressed as

(1.11) $\left\|R_{h} u-u\right\|_{-q} \leq C h^{p+q}\|u\|_{p}$ for $u \in H^{p} \cap H_{0}^{1},-1 \leq q \leq r-2,2 \leq p \leq r$.

Throughout this paper, $C$ will denote, as above, a positive constant independent of $h$ and the functions involved, not necessarily the same at different occurrences.

2. Error Estimates in the Case of a Smooth Solution. This section is concerned with the following error estimates for the semidiscrete finite element method (1.3) in the case that the continuous problem (1.1) has a smooth solution.

THEOREM 2.1. Let $u$ and $u_{h}$ be the solutions of (1.1) and (1.3), respectively. Then we have

$$
\left\|u_{h}(t)-u(t)\right\| \leq C\left\|v_{h}-v\right\|+C h^{r}\left\{\|v\|_{r}+\int_{0}^{t}\left\|u_{t}\right\|_{r} d s\right\} \quad \text { for } t \in \bar{J} .
$$

Proof. Following Wheeler [8], we write the error, with $R_{h}$ defined by (1.10), as

$$
e=u_{h}-u=\left(u_{h}-R_{h} u\right)+\left(R_{h} u-u\right)=\theta+\rho .
$$

Here we have at once from (1.11)

$$
\|\rho(t)\| \leq C h^{r}\|u(t)\|_{r} \leq C h^{r}\left\{\|v\|_{r}+\int_{0}^{t}\left\|u_{t}\right\|_{r} d s\right\} \quad \text { for } t \in \bar{J} .
$$

We continue to estimate $\theta=u_{h}-R_{h} u \in S_{h}$. We find easily by (1.1), (1.3) and (1.10) that

$$
\left(\theta_{t}, \chi\right)+A(\theta, \chi)=-\left(\rho_{t}, \chi\right)+\tilde{B}(e, \chi) \quad \forall \chi \in S_{h}, t \in J .
$$

We now write $\theta=\theta^{1}+\theta^{2}$, where $\theta^{1}$ and $\theta^{2}: \bar{J} \rightarrow S_{h}$ are determined by

$$
\begin{aligned}
& \left(\theta_{t}^{1}, \chi\right)+A\left(\theta^{1}, \chi\right)=-\left(\rho_{t}, \chi\right) \quad \forall \chi \in S_{h}, t \in J, \\
& \theta^{1}(0)=\theta(0),
\end{aligned}
$$


and

$$
\begin{aligned}
& \left(\theta_{t}^{2}, \chi\right)+A\left(\theta^{2}, \chi\right)=\tilde{B}(e, \chi) \quad \forall \chi \in S_{h}, t \in J, \\
& \theta^{2}(0)=0 .
\end{aligned}
$$

Here the standard argument for the differential equation shows (cf. [7, Chapter 1])

$$
\left\|\theta^{1}(t)\right\| \leq C\left\|v_{h}-v\right\|+C h^{r}\left\{\|v\|_{r}+\int_{0}^{t}\left\|u_{t}\right\|_{r} d s\right\} \quad \text { for } t \in \bar{J}
$$

and we are left with estimating $\theta^{2}$.

Setting $\chi=T_{h} \theta_{t}^{2}$ in (2.3), we find

$$
\begin{aligned}
\left(T_{h} \theta_{t}^{2}, \theta_{t}^{2}\right)+\frac{1}{2} \frac{d}{d t}\left\|\theta^{2}(t)\right\|^{2}=\int_{0}^{t} B\left(t, s ; e(s), T_{h} \theta_{t}^{2}(t)\right) d s \\
=\frac{d}{d t} \int_{0}^{t} B\left(t, s ; e(s), T_{h} \theta^{2}(t)\right) d s-B\left(t, t ; e(t), T_{h} \theta^{2}(t)\right) \\
\quad-\int_{0}^{t} B_{t}\left(t, s ; e(s), T_{h} \theta^{2}(t)\right) d s
\end{aligned}
$$

where $B_{t}$ corresponds to the operator (of order $\beta$ ) obtained by differentiating the coefficients of $B$ with respect to $t$. Hence, by integration with respect to $t$, we obtain

$$
\begin{aligned}
\left\|\theta^{2}(t)\right\|^{2} \leq & C \int_{0}^{t}\left\{\left|B\left(t, s ; e(s), T_{h} \theta^{2}(t)\right)\right|+\left|B\left(s, s ; e(s), T_{h} \theta^{2}(s)\right)\right|\right\} d s \\
& +C \int_{0}^{t} \int_{0}^{s}\left|B_{t}\left(s, \tau ; e(\tau), T_{h} \theta^{2}(s)\right)\right| d \tau d s \equiv Q(t) \quad \text { for } t \in \bar{J} .
\end{aligned}
$$

We shall prove that the quantity $Q(t)$ thus defined satisfies

$$
Q(t) \leq C\left\{\left\|v_{h}-v\right\|+h^{r}\left(\|v\|_{r}+\int_{0}^{t}\left\|u_{t}\right\|_{r} d s\right)+\int_{0}^{t}\|e\| d s\right\} \sup _{s \leq t}\left\|\theta^{2}(s)\right\| .
$$

Assuming this for a moment, we find easily from (2.6) that

$$
\left\|\theta^{2}(t)\right\| \leq C\left\{\left\|v_{h}-v\right\|+h^{r}\left(\|v\|_{r}+\int_{0}^{t}\left\|u_{t}\right\|_{r} d s\right)+\int_{0}^{t}\|e\| d s\right\} .
$$

Combining this with (2.1) and (2.4), we derive

$$
\begin{aligned}
\|e(t)\| & \leq\|\rho(t)\|+\left\|\theta^{1}(t)\right\|+\left\|\theta^{2}(t)\right\| \\
& \leq C\left\|v_{h}-v\right\|+C h^{r}\left\{\|v\|_{r}+\int_{0}^{t}\left\|u_{t}\right\|_{r} d s\right\}+C \int_{0}^{t}\|e\| d s .
\end{aligned}
$$

An application of Gronwall's lemma now completes the proof.

It remains to prove (2.7). For this purpose we need the following

LEMMA 2.1. Let $B(t, s ; \cdot, \cdot)$ be a bilinear form associated with a partial differential operator $B(t, s)$ of order $\beta \leq 2$. Then

$$
\left|B\left(t, s ; f, T_{h} g\right)\right| \leq C\left(h\|f\|_{1}+\|f\|\right)\|g\| \quad \text { for } 0 \leq s \leq t \in \bar{J}, f \in H_{0}^{1}, g \in L_{2} .
$$

Proof. By (1.8) and (1.9) we have, with $B^{*}$ the adjoint of $B$,

$$
\begin{aligned}
\left|B\left(t, s ; f, T_{h} g\right)\right| & \leq\left|B\left(t, s ; f,\left(T_{h}-T\right) g\right)\right|+|B(t, s ; f, T g)| \\
& \leq C\|f\|_{1}\left\|\left(T_{h}-T\right) g\right\|_{1}+\|f\|\left\|B(t, s)^{*} T g\right\| \\
& \leq C\left(h\|f\|_{1}+\|f\|\right)\|g\| . \quad \square
\end{aligned}
$$


We now return to the proof of (2.7). By Lemma 2.1 we obtain easily

$$
Q(t) \leq C \int_{0}^{t}\left(h\|e\|_{1}+\|e\|\right) d s \sup _{s \leq t}\left\|\theta^{2}(s)\right\|
$$

and the proof of (2.7) can hence be completed by showing that

$$
\int_{0}^{t}\|e\|_{1} d s \leq C\left\|v_{h}-v\right\|+C h^{r-1}\left(\|v\|_{r}+\int_{0}^{t}\left\|u_{t}\right\|_{r} d s\right) .
$$

Inserting $\chi=\theta=\theta(t)$ into the error identity (2.2), we obtain

$$
\begin{aligned}
\frac{1}{2} \frac{d}{d t}\|\theta\|^{2}+A(\theta, \theta) & =-\left(\rho_{t}, \theta\right)+\int_{0}^{t} B(t, s ; e(s), \theta(t)) d s \\
& \leq\left\|\rho_{t}\right\|\|\theta\|+C \int_{0}^{t}\|e\|_{1} d s\|\theta\|_{1} \\
& \leq\left\|\rho_{t}\right\|\|\theta\|+C\left(\int_{0}^{t}\|e\|_{1} d s\right)^{2}+\frac{1}{2} A(\theta, \theta) .
\end{aligned}
$$

Therefore, we have after integration in time,

$$
\begin{aligned}
\|\theta(t)\|^{2}+\int_{0}^{t}\|\theta\|_{1}^{2} d s \leq & C\|\theta(0)\|^{2}+C \int_{0}^{t}\left\|\rho_{t}\right\|\|\theta\| d s+C \int_{0}^{t}\left(\int_{0}^{s}\|e\|_{1} d \tau\right)^{2} d s \\
\leq & C\|\theta(0)\|^{2}+C\left(\int_{0}^{t}\left\|\rho_{t}\right\| d s\right)^{2}+\frac{1}{2} \sup _{s \leq t}\|\theta(s)\|^{2} \\
& +C \int_{0}^{t}\left(\int_{0}^{s}\|e\|_{1} d \tau\right)^{2} d s .
\end{aligned}
$$

Since the above inequality is valid for all $t \in \bar{J}$, we obtain

$$
\begin{aligned}
\sup _{s \leq t}\|\theta(s)\|^{2}+\int_{0}^{t}\|\theta\|_{1}^{2} d s \leq & C\|\theta(0)\|^{2}+C\left(\int_{0}^{t}\left\|\rho_{t}\right\| d s\right)^{2} \\
& +C \int_{0}^{t}\left(\int_{0}^{s}\|e\|_{1} d \tau\right)^{2} d s
\end{aligned}
$$

Now, recalling that the interval $J$ is bounded, we find

$$
\begin{aligned}
\left(\int_{0}^{t}\|e\|_{1} d s\right)^{2} & \leq C \int_{0}^{t}\|\theta\|_{1}^{2} d s+C\left(\int_{0}^{t}\|\rho\|_{1} d s\right)^{2} \\
& \leq C \int_{0}^{t}\|\theta\|_{1}^{2} d s+C\left(h^{r-1} \int_{0}^{t}\|u\|_{r} d s\right)^{2}
\end{aligned}
$$

and hence, using (2.11) for the first term on the right and the standard estimate for $\rho$, yields

$$
\begin{aligned}
\left(\int_{0}^{t}\|e\|_{1} d s\right)^{2} \leq & C\left\|v_{h}-v\right\|^{2}+C h^{2(r-1)}\left(\|v\|_{r}^{2}+\int_{0}^{t}\left\|u_{t}\right\|_{r} d s\right)^{2} \\
& +C \int_{0}^{t}\left(\int_{0}^{s}\|e\|_{1} d \tau\right)^{2} d s
\end{aligned}
$$

whence (2.10) follows by using Gronwall's lemma. 
Remark 2.1. The difficult case in the proof is $\beta=2$. When $\beta \leq 1$, we have

$$
|B(t, s ; e, \chi)| \leq C\|e\|\|\chi\|_{1},
$$

and hence easily from (2.3), with $\chi=\theta^{2}(t)$,

$$
\left\|\theta^{2}(t)\right\| \leq C \int_{0}^{t}\|e(s)\| d s
$$

which implies (2.8) more directly. Also, when $\beta=2$, the proof of (2.7) from (2.9) follows in a straightforward way directly in the presence of the inverse assumption $\|\chi\|_{1} \leq C h^{-1}\|\chi\|$ for $\chi \in S_{h}$.

Remark 2.2. The part of the standard approximability assumption (1.2) which concerns the gradient of $u$ is somewhat difficult to satisfy in practice when $r>2$. However, Theorem 2.1 remains valid if (1.2) is replaced by

$$
\inf _{\chi \in S_{h}}\left\{\|u-\chi\|+h\|u-\chi\|_{1, \Omega_{h}}\right\} \leq C h^{s}\|u\|_{s} \text { for } 1 \leq s \leq r, \text { if } u \in H^{s} \cap H_{0}^{1},
$$

where $\Omega_{h} \subseteq \Omega$ is a mesh domain with $\sup _{x \in \Omega \backslash \Omega_{h}} \operatorname{dist}(x, \partial \Omega) \leq c h^{r}$ such that the elements of $S_{h}$ vanish in $\Omega \backslash \Omega_{h}$, and where $\|\cdot\|_{1, \Omega_{h}}$ is the norm in $H^{1}\left(\Omega_{h}\right)$. This assumption holds for (carefully constructed) isoparametric finite element spaces. The modification of the proof consists in using the error estimates for $R_{h}$ corresponding to (2.13), together with the inequality

$$
\|v\|_{1, \Omega \backslash \Omega_{h}} \leq C h^{r / 2}\|v\|_{2}
$$

to show that (2.9) may now be replaced by

$$
Q(t) \leq C \int_{0}^{t}\left(h\|e\|_{1, \Omega_{h}}+\|e\|+h^{r}\|u\|_{2}\right) d s \sup _{s \leq t}\left\|\theta^{2}(s)\right\|,
$$

and in substituting $\|\cdot\|_{1, \Omega_{h}}$ for $\|\cdot\|_{1}$ in the subsequent arguments. Using (2.12) for $\beta \leq 1$, it is seen that the change in the proof is only needed when $\beta=2$.

3. The Homogeneous Equation with Nonsmooth Data. In this section we shall discuss the regularity of the solution of (1.1) in the case that the PIDE is homogeneous (i.e., when $f=0$ ) and $v$ is only in $L_{2}$.

By Duhamel's principle, we may formally write (1.1) with $f=0$ in the form

$$
u(t)=E(t) v+\int_{0}^{t} E(t-s) \tilde{B} u(s) d s \quad \text { for } t \in \bar{J} .
$$

For our present purpose we shall consider $u$ to be a solution of (1.1) with $f=0$ if $u \in C\left(J ; \dot{H}^{2}\right) \cap C\left(\bar{J} ; L_{2}\right)$ and satisfies (3.1). Here and below, $C(J ; H)$ denotes the continuous functions in $J$ with values in the Hilbert space $H$, and similarly for $C(\bar{J} ; H)$. We note, in particular, that when $u \in C(\bar{J} ; H)$, the $H$-norm of $u(t)$ is bounded on $J$, whereas this is not necessarily the case if $u \in C(J ; H)$.

We shall prove the following result.

THEOREM 3.1. For $v \in L_{2}$, the equation (3.1) admits a unique solution $u$, which belongs to $C\left(J ; \dot{H}^{4-\beta}\right)$ for $\beta=0$ and 2 , and to $C\left(J ; H^{3} \cap \dot{H}^{2}\right)$ for $\beta=1$. Furthermore,

$$
\|u(t)\|_{\alpha} \leq C t^{-\alpha / 2}\|v\| \quad \text { for } t \in J, 0 \leq \alpha \leq 4-\beta
$$


We reiterate that this result shows the same smoothness property as for the purely parabolic equation when $\alpha \leq 4-\beta$. As we shall demonstrate by counterexamples at the end of this section, this limit for $\alpha$ is sharp.

Denoting the integral in (3.1) by $w(t)$, we find, since $u(t)=E(t) v+w(t)$,

$$
\begin{aligned}
w(t) & =\int_{0}^{t} E(t-s) \tilde{B} u(s) d s \\
& =\int_{0}^{t} E(t-s) \tilde{B} E v(s) d s+\int_{0}^{t} E(t-s) \tilde{B} w(s) d s \\
& \equiv V(t)+K w(t),
\end{aligned}
$$

where $\tilde{B} E v$ is defined by (1.1) with $u(s)$ replaced by $E(s) v$. We shall prove that for $v \in L_{2}$ this Volterra-type integral equation in $w$ has a unique solution $w(t) \in$ $C\left(\bar{J} ; \dot{H}^{4-\beta}\right)$ for $\beta=0$ and 2 , and $w(t) \in C\left(\bar{J} ; H^{3} \cap \dot{H}^{2}\right)$ for $\beta=1$, and that

$$
\|w(t)\|_{4-\beta} \leq C\|v\| \text { for } t \in \bar{J} \text {. }
$$

In view of the well-known estimate (1.6) for $E(t) v$, this will show Theorem 3.1. Note in particular that the term $w(t)$ does not have the singular behavior of $E(t) v$ at $t=0$.

For the purpose of the proof we shall analyze the two terms on the right in (3.2). We begin with the following lemma, where we note the alternative uses of the norms in $\dot{H}^{s}$ and $H^{s}$. This is motivated by subsequent applications to functions satisfying and not satisfying boundary conditions, respectively, the latter case occurring for functions of the form $B u$ when $\beta=1$.

LEMMA 3.1. Let $\alpha \geq 0$. Under the appropriate regularity assumptions for $g$, we have

$$
\left|\int_{0}^{t} E(t-s) g(s) d s\right|_{\alpha+2} \leq C \sup _{s \leq t}\left(|g(s)|_{\alpha}+s\left|g^{\prime}(s)\right|_{\alpha}\right) \quad \text { for } t \in \bar{J}
$$

and

$$
\begin{aligned}
\left\|\int_{0}^{t} E(t-s) g(s) d s\right\|_{\alpha+3} \leq & C \sup _{s \leq t}\|g(s)\|_{\alpha+1} \\
& +C t^{-1 / 2} \sup _{s \leq t}\left(|g(s)|_{\alpha}+s\left|g^{\prime}(s)\right|_{\alpha}\right) \quad \text { for } t \in J .
\end{aligned}
$$

Proof. Using integration by parts in the second term on the right, we find, since $\frac{d}{d s} T E(s)=-E(s)$,

$$
\begin{array}{rl}
t \int_{0}^{t} & E(t-s) g(s) d s=\int_{0}^{t}(t-s) E(t-s) g(s) d s+\int_{0}^{t} s E(t-s) g(s) d s \\
& =t T g(t)+\int_{0}^{t}\left\{((t-s) I-T) E(t-s) g(s)-s T E(t-s) g^{\prime}(s)\right\} d s \\
& \equiv t T g(t)+t G(t) .
\end{array}
$$

For the $G(t)$ thus defined, we obtain by the boundedness of $T: \dot{H}^{\alpha+\delta} \rightarrow \dot{H}^{\alpha+2+\delta}$ and by (1.6) that

$$
\begin{aligned}
|G(t)|_{\alpha+2+\delta} & \leq C t^{-1} \int_{0}^{t}(t-s)^{-\delta / 2}\left(|g(s)|_{\alpha}+s\left|g^{\prime}(s)\right|_{\alpha}\right) d s \\
& \leq C t^{-\delta / 2} \sup _{s \leq t}\left(|g(s)|_{\alpha}+s\left|g^{\prime}(s)\right|_{\alpha}\right) \quad \text { for } t \in J, \delta=0,1 .
\end{aligned}
$$


The desired inequalities now follow, since $T: \dot{H}^{\alpha} \rightarrow \dot{H}^{\alpha+2}$ and $T: H^{\alpha+1} \rightarrow H^{\alpha+3}$ are bounded.

In order to derive the required estimate for the term $V(t)$ in (3.2) we now show:

LEMMA 3.2. Let $B(t, \varepsilon)$ be a partial differential operator of order $\beta \leq 2$. Then $v \in L_{2}$ implies that $\tilde{B} E v \in C\left(\bar{J} ; H^{2-\beta}\right)$. If in particular, $\beta=0$, then $\tilde{B} E v \in$ $C\left(\bar{J} ; \dot{H}^{2}\right)$. Furthermore,

$$
\|\tilde{B} E v(t)\|_{2-\beta} \leq C\|v\| \quad \text { for } t \in \bar{J}
$$

and

$$
\|\tilde{B} E v(t)\| \leq C t^{1-\beta / 2}\|v\| \quad \text { for } t \in \bar{J}
$$

Proof. We obtain by integration by parts

$$
\tilde{B} E v(t)=\int_{0}^{t} B(t, s) E(s) v d s=-B(t, t) T E(t) v+B(t, 0) T v+\tilde{B}_{s} T E v(t)
$$

where $\tilde{B}_{s}$ is defined in terms of $B_{s}=(\partial / \partial s) B$. Hence, by the boundedness of $T: L_{2} \rightarrow H^{2}$, we find

$$
\|\tilde{B} E v(t)\|_{2-\beta} \leq C \sup _{s \leq t}\|T E(s) v\|_{2} \leq C\|v\| .
$$

When $\beta=0$ and $\beta=1$,

$$
\|\tilde{B} E v(t)\| \leq C \int_{0}^{t}\|E(s) v\|_{\beta} d s \leq C \int_{0}^{t} s^{-\beta / 2} d s\|v\| \leq C t^{1-\beta / 2}\|v\| .
$$

For $\beta=0, B(t, s)$ is a multiplication by a scalar function and hence $\tilde{B} E v(t) \in \dot{H}^{2}$ for $t \in \bar{J}$.

We are now ready to derive our estimate for the term $V(t)$ in (3.2).

LEMMA 3.3. Assuming $v \in L_{2}$, we have $V \in C\left(\bar{J} ; \dot{H}^{4-\beta}\right)$ for $\beta=0$ and 2 , and $V \in C\left(\bar{J} ; H^{3} \cap \dot{H}^{2}\right)$ for $\beta=1$. Further,

$$
\|V(t)\|_{4-\beta} \leq C\|v\| \quad \text { for } t \in \bar{J}, \beta \leq 2 .
$$

Proof. Considering first $\beta=0$ and 2, we have by (3.3)

$$
|V(t)|_{4-\beta} \leq C \sup _{s \leq t}\left(|\tilde{B} E v(s)|_{2-\beta}+s\left|(\tilde{B} E v)^{\prime}(s)\right|_{2-\beta}\right) .
$$

The first term on the right is estimated directly by Lemma 3.2, and for the second we have, since $(\tilde{B} E v)^{\prime}(s)=B(s, s) E(s) v+\tilde{B}_{t} E v(s)$ and applying now also (1.6) and Lemma 3.2 to $B_{s}$, that

$$
s\left|(\tilde{B} E v)^{\prime}(s)\right|_{2-\beta} \leq C\left(s|E(s) v|_{2}+s\left|\tilde{B}_{t} E v(s)\right|_{2-\beta}\right) \leq C\|v\| .
$$

Here we have used the fact that for $\beta=0$ the operators $B$ and $B_{t}$ consist of multiplication by a scalar function and are thus bounded in $\dot{H}^{2}$.

In the case $\beta=1$ we obtain similarly, using instead (3.4) and Lemma 3.2,

$$
\|V(t)\|_{3} \leq C \sup _{s \leq t}\|\tilde{B} E v(s)\|_{1}+C t^{-1 / 2} \sup _{s \leq t}\left(\|\tilde{B} E v(s)\|+s\left\|(\tilde{B} E v)^{\prime}(s)\right\|\right) \leq C\|v\| .
$$

The fact that $V \in C\left(\bar{J} ; \dot{H}^{2}\right)$ follows from (3.3) of Lemma 3.1 with $\alpha=0$. The proof is now complete.

The following lemma is concerned with the properties of the operator $K$ defined in (3.2). 
LEMMA 3.4. The operator $K$ is bounded in $C\left(\bar{J} ; \dot{H}^{2}\right)$ and

$$
|K g(t)|_{2} \leq C \int_{0}^{t}|g(s)|_{2} d s \quad \text { for } t \in \bar{J} .
$$

Furthermore, for $g \in C\left(\bar{J} ; \dot{H}^{2}\right)$, we have $K g(t) \in C\left(\bar{J} ; \dot{H}^{4}\right)$ for $\beta=0$ and $K g(t) \in$ $C\left(\bar{J} ; H^{3} \cap \dot{H}^{2}\right)$ for $\beta=1$, and

$$
\|K g(t)\|_{4-\beta} \leq C \sup _{s \leq t}|g(s)|_{2} \quad \text { for } t \in \bar{J}, \beta \leq 2 .
$$

Proof. Replacing $g$ by $\tilde{B} g$ in (3.5), we obtain

$$
|K g(t)|_{2} \leq C \sup _{s \leq t}\|\tilde{B} g(s)\|+C \int_{0}^{t}\left\|(\tilde{B} g)^{\prime}(s)\right\| d s \leq C \int_{0}^{t}|g(s)|_{2} d s
$$

which shows (3.6) and the case $\beta=2$ of (3.7). For $\beta=0$, we have by (3.3)

$$
|K g(t)|_{4} \leq C \sup _{s \leq t}\left(|\tilde{B} g(s)|_{2}+s\left|(\tilde{B} g)^{\prime}(s)\right|_{2}\right) \leq C \sup _{s \leq t}|g(s)|_{2}
$$

and for $\beta=1$, by (3.4),

$$
\begin{aligned}
\|K g(t)\|_{3} & \leq C \sup _{s \leq t}\|\tilde{B} g(s)\|_{1}+C t^{-1 / 2} \sup _{s \leq t}\left(\|\tilde{B} g(s)\|+s\left\|(\tilde{B} g)^{\prime}(s)\right\|\right) \\
& \leq C \sup _{s \leq t}|g(s)|_{2} .
\end{aligned}
$$

This completes the proof.

Proof of Theorem 3.1. Consider the Volterra type equation $w=V+K w$ for $w$. Since by Lemma $3.4, K$ is a bounded operator in $C\left(\bar{J} ; \dot{H}^{2}\right)$ which satisfies (3.6), and since $V \in C\left(\bar{J} ; \dot{H}^{2}\right)$ by Lemma 3.3 , we conclude by the standard argument for Volterra equations that this equation has a unique solution $w \in C\left(\bar{J} ; \dot{H}^{2}\right)$, and

$$
|w(t)|_{2} \leq C \sup _{s \leq t}|V(t)|_{2} \leq C\|v\| .
$$

The regularity statements for $w$ now follow by Lemmas 3.3 and 3.4, since

$$
\|w(t)\|_{4-\beta} \leq\|V(t)\|_{4-\beta}+\|K w(t)\|_{4-\beta} \leq C\|v\|+C \sup _{s \leq t}|w(s)|_{2} \leq C\|v\| .
$$

In view of our above discussion this completes the proof of Theorem 3.1.

The following result will be used in Section 4 .

LEMMA 3.5. Let $u(t)$ be the solution of (3.1) with $v \in L_{2}$. Then

$$
\left\|\tilde{B}_{t} u(t)\right\|_{2-\beta}+\|\tilde{B} u(t)\|_{2-\beta} \leq C\|v\| \quad \text { for } t \in \bar{J}
$$

and

$$
\left\|\tilde{B}_{t} u(t)\right\|+\|\tilde{B} u(t)\| \leq C t^{1-\beta / 2}\|v\| \quad \text { for } t \in J .
$$

Proof. Since $\tilde{B} u=\tilde{B} E v+\tilde{B} w$, the bounds for $\tilde{B} u$ follow easily from Lemma 3.2 and (3.8). Since the arguments apply equally well to $\tilde{B}_{t}$, the lemma is established.

We shall now demonstrate that the result of Theorem 3.1 is sharp in the sense that, for general $B$ of order $\beta$, higher-order regularity than $H^{4-\beta}$ cannot be attained for $v$ only in $L_{2}$. We shall do this by exhibiting one PIDE for each of the cases of 
$\beta=0,1$, and 2 , with the property that if $u(t) \in H^{\alpha}$ for some $\alpha>4-\beta$ and some $t \in J$, then $v$ must belong to a space $H^{s}$ with $s$ positive.

We consider first the equation (3.1) with $B=I$ and prove that then $u(t)-T^{2} v \in$ $\dot{H}^{5}$ for $t \in J$. From this we may conclude that, if $u(t) \in H^{\alpha}$ with $4<\alpha \leq 5$ and $t \in J$, then $T^{2} v \in H^{\alpha} \cap \dot{H}^{4}$, so that $v \in H^{\alpha-4}$, which shows our claim for $\beta=0$.

Using our above notation, we have by (1.6) and (3.8), noting that $w \in \dot{H}^{4}$ for $t \in J$, that

$$
|K w(t)|_{5} \leq C \int_{0}^{t}(t-s)^{-1 / 2} \int_{0}^{s}|w(\tau)|_{4} d \tau d s \leq C\|v\| \quad \text { for } t \in J
$$

and hence that $K w(t) \in \dot{H}^{5}$ for $t \in J$. Further,

$$
V(t)=\int_{0}^{t} E(t-s) \int_{0}^{s} E(\tau) v d \tau d s=T^{2} v-T^{2} E(t) v-t T E(t) v,
$$

and since the last two terms are smooth for any positive $t$, this shows that $w(t)-$ $T^{2} v=V(t)-T^{2} v+K w(t) \in \dot{H}^{5}$. Since $u(t)-w(t)=E(t) v \in \dot{H}^{5}$ for any positive $t$, this implies $u(t)-T^{2} v \in \dot{H}^{5}$ for $t \in J$ and thus completes the proof.

We next consider $\beta=1$ and choose $B=D_{1} \equiv \partial / \partial x_{1}$. We shall now show $u(t)-T D_{1} T v \in H^{\alpha} \cap \dot{H}^{2}$ for $t \in J$ and any $\alpha<4$, from which we shall conclude as before that no higher regularity than $u(t) \in H^{3}$ holds for all $v \in L_{2}$, thus confirming the sharpness in this case. In fact, if $u(t) \in H^{\alpha}$ with $3<\alpha<4$, then we would have $D_{1} T v \in H^{\alpha-2}$, which is not true for all $v \in L_{2}$, since $D_{1} \psi$ is not in $H^{\alpha-2}$ for all $\psi \in \dot{H}^{2}$ when $\alpha-2>1$.

Here we know that $w \in C\left(\bar{J} ; H^{3} \cap \dot{H}^{2}\right)$ and hence $T D_{1} w \in C\left(\bar{J} ; H^{4} \cap \dot{H}^{2}\right)$, so that $E(t-\cdot) T D_{1} w \in L_{1}\left((0, t) ; \dot{H}^{\alpha}\right)$, uniformly in $t$, for any $\alpha<4$, where $L_{1}(J ; H)$ denotes the set of functions $J \rightarrow H$ with $H$-norm integrable over $J$. Thus,

$$
K w(t)=\int_{0}^{t}(I-E(t-\tau)) T D_{1} w(\tau) d \tau \in H^{\alpha} \cap \dot{H}^{2} \quad \text { for } t \in \bar{J} .
$$

This time,

$$
\begin{aligned}
V(t) & =\int_{0}^{t} E(t-s) \int_{0}^{s} D_{1} E(\tau) v d \tau d s \\
& =T D_{1} T v-T D_{1} T E(t) v-\int_{0}^{t} T E(t-s) D_{1} E(s) v d s
\end{aligned}
$$

where the last two terms are both in $H^{\alpha} \cap \dot{H}^{2}$ for $t \in J$. This shows $u(t)-T D_{1} T v=$ $E(t) v+w(t)-T D_{1} T v \in H^{\alpha} \cap \dot{H}^{2}$ for $t \in J$ and completes the proof.

We finally consider $\beta=2$ and now choose $B=A$. This time we shall show $u(t)-e^{t} T v \in \dot{H}^{3}$ for $t \in J$, from which we infer that $u(t) \in \dot{H}^{2}$ is the highest regularity valid for all $v \in L_{2}$.

We now have $w \in C\left(\bar{J} ; \dot{H}^{2}\right)$ and

$$
K w(t)=\int_{0}^{t} w(s) d s-\int_{0}^{t} E(t-s) w(s) d s,
$$

where the second integral is in $C\left(\bar{J} ; \dot{H}^{3}\right)$, because $E(t-\cdot) w \in L_{1}\left((0, t) ; \dot{H}^{3}\right)$, uniformly in $t$. Further, $V(t)=T v-T E(t) v-t E(t) v$, so that $w$ is of the form

$$
w(t)=T v+g(t)+\int_{0}^{t} w(s) d s, \quad \text { with } g \in L_{1}\left(J ; \dot{H}^{3}\right) \cap C\left(J ; \dot{H}^{3}\right) .
$$


Hence,

$$
w(t)=T v+g(t)+\int_{0}^{t} e^{t-s}(T v+g(s)) d s=e^{t} T v+h(t),
$$

where $h(t) \in \dot{H}^{3}$ for $t \in J$. By the regularity of $u(t)-w(t)=E(t) v$, this completes the proof.

4. Error Estimates for the Homogeneous Equation with Nonsmooth Data. In this section we shall prove the following nonsmooth data error estimate for the spatially discrete finite element method for our homogeneous PIDE.

THEOREM 4.1. Let $u$ be the solution of (1.1) with $f=0$ and $v \in L_{2}$, where $B(t, s)$ is a partial differential operator of order $\beta, \beta \leq 2$. Let further $u_{h}$ be the solution of the corresponding semidiscrete problem (1.3) with $f=0$ and $v_{h}=P_{h} v$. Then we have

$$
\left\|u_{h}(t)-u(t)\right\| \leq C h^{\gamma} t^{-\gamma / 2}\|v\| \quad \text { for } t \in J, \text { where } \gamma=\min (4-\beta, r) .
$$

Clearly, in view of Theorem 3.1, the power of $h$ occurring in this estimate is best possible.

In the proof we may, and shall, assume that $4-\beta \leq r$, so that $\gamma=4-\beta$. In fact, if $r=2$ or 3 and $\beta<4-r$, then we may interpret $B$ to be of order $4-r$, and the results in this case will lead to the correct conclusion.

Defining the discrete analogue $B_{h}=B_{h}(t, s): S_{h} \rightarrow S_{h}$ of $B=B(t, s)$ by

$$
\left(B_{h}(t, s) \psi, \chi\right)=B(t, s ; \psi, \chi) \quad \forall \psi, \chi \in S_{h}, 0 \leq s \leq t \in \bar{J}
$$

we write the semidiscrete problem (1.3) with $f=0$ in the form

$$
\begin{aligned}
& u_{h, t}+A_{h} u_{h}=\int_{0}^{t} B_{h}(t, s) u_{h}(s) d s \equiv \tilde{B}_{h} u_{h}(t) \quad \text { for } t \in J, \\
& u_{h}(0)=P_{h} v .
\end{aligned}
$$

By Duhamel's principle we then have for the semidiscrete solution

$$
u_{h}(t)=E_{h}(t) P_{h} v+\int_{0}^{t} E_{h}(t-s) \tilde{B}_{h} u_{h}(s) d s .
$$

Together with (3.1), this shows for the error $e=u_{h}-u$ that

$$
\begin{aligned}
e(t)= & \left(E_{h}(t) P_{h}-E(t)\right) v+\int_{0}^{t} E_{h}(t-s) \tilde{B}_{h} u_{h}(s) d s \\
& -\int_{0}^{t} E(t-s) \tilde{B} u(s) d s \\
= & F_{h}(t) v+\int_{0}^{t} F_{h}(t-s) \tilde{B} u(s) d s \\
& +\int_{0}^{t} E_{h}(t-s)\left(\tilde{B}_{h} u_{h}(s)-P_{h} \tilde{B} u(s)\right) d s \\
\equiv & e_{0}(t)+e_{1}(t)+e_{2}(t) \equiv e_{0}(t)+\hat{e}(t) .
\end{aligned}
$$

We shall prove below, by estimating $e_{1}(t)$ and $e_{2}(t)$ separately, that

$$
\|\hat{e}(t)\| \leq C h^{4-\beta}\|v\| \text {. }
$$


Together with the known estimate (1.7) for $e_{0}(t)=F_{h}(t) v$, the error for the finite element solution of the associated differential equation problem, this will complete the proof. We remark that, analogously to the integral term in (3.1), the contribution of $\hat{e}$ to the error thus does not exhibit any singularity at $t=0$.

The proof will be based on a sequence of lemmas. In the first one we study the selfadjoint operator $H_{h}(t): L_{2} \rightarrow H_{0}^{1}$ defined by

$$
H_{h}(t)=E_{h}(t) T_{h}-E(t) T \text {. }
$$

This operator is a time integral of $-F_{h}(t)$ and is introduced to avoid the singular behavior of $F_{h}(t)$ at $t=0$.

LEMMA 4.1. We have

$$
\left|H_{h}(t) v\right|_{-q} \leq C h^{p} t^{1-(p-q) / 2}\|v\| \quad \text { for } t \in J, 1 \leq q+2 \leq p \leq r,
$$

and

$$
\left\|H_{h}(t) v\right\| \leq C h^{4}|v|_{2} \quad \text { for } t \in \bar{J}, r \geq 4 .
$$

Proof. We may write

$$
H_{h}(t)=T_{h} F_{h}(t)+\left(T_{h}-T\right) E(t) .
$$

Since by (1.6) and (1.8), the last term above may be bounded as desired, we need only consider the first term on the right. We shall now appeal to the analysis used in [7, Chapter 6], and estimate $e_{0}(t)=F_{h}(t) v$ in the appropriate discrete seminorm defined by $|v|_{-s, h}=\left(T_{h}^{s} v, v\right)^{1 / 2}$ (for $v \in S_{h}$ also for $s=-1$ ). We start by proving (4.3). Using Lemma 3 of [7, Chapter 6] we obtain

$$
\begin{aligned}
\left|T_{h} e_{0}\right|_{-q} & \leq C\left|e_{0}\right|_{-(q+2), h}+C h^{q}\left|e_{0}\right|_{-2, h} \\
& \leq C\left|e_{0}\right|_{-(q+2)}+C h^{q}\left|e_{0}\right|_{-2}+C h^{q+2}\left\|e_{0}\right\| \quad \text { for } 0 \leq q \leq r-2,
\end{aligned}
$$

and, for $q=-1$,

$$
\left|T_{h} e_{0}\right|_{1}=\left|e_{0}\right|_{-1, h} \leq C\left(\left|e_{0}\right|_{-1}+h\left\|e_{0}\right\|\right) .
$$

For any $\varphi \in \dot{H}^{i}$ we have by (1.7) that

$$
\left|\left(e_{0}, \varphi\right)\right|=\left|\left(v, F_{h}(t) \varphi\right)\right| \leq C h^{j} t^{-(j-i) / 2}\|v\||\varphi|_{i} \quad \text { for } 0 \leq i \leq j \leq r,
$$

whence

$$
\left|e_{0}(t)\right|_{-i} \leq C h^{j} t^{-(j-i) / 2}\|v\| \text { for } 0 \leq i \leq j \leq r .
$$

Together with (4.5), (4.6) and (1.7), this shows

$$
\left|T_{h} F_{h}(t) v\right|_{-q} \leq C h^{p} t^{1-(p-q) / 2}\|v\| \quad \text { for } 1 \leq q+2 \leq p \leq r,
$$

which completes the proof of (4.3).

For the proof of the corresponding part of (4.4) we note that

$$
T_{h} e_{0, t}+e_{0}=\rho_{0} \equiv-\left(R_{h}-I\right) E(t) v,
$$

and hence, by Lemma 4 of [7, Chapter 3], Lemma 3 of [7, Chapter 6], (1.8) and (1.6), that for $r \geq 4$

$$
\left\|T_{h} e_{0}\right\|=\left|e_{0}\right|_{-2, h} \leq C \sup _{s \leq t}\left(s\left|\rho_{0}^{\prime}(s)\right|_{-2, h}+\left|\rho_{0}(s)\right|_{-2, h}\right) \leq C h^{4}|v|_{2} .
$$


In our next lemma we shall use the notation

$$
\tilde{F}_{h} g(t)=\int_{0}^{t} F_{h}(t-s) g(s) d s \quad \text { for } t \in \bar{J}, g \in C\left(\bar{J} ; L_{2}\right) .
$$

LEMMA 4.2. Under the appropriate regularity assumptions for $g$, we have

$$
\begin{aligned}
& \left\|\tilde{F}_{h} g(t)\right\| \leq C h^{p+2} \sup _{s \leq t}\left(|g(s)|_{p}+s\left|g^{\prime}(s)\right|_{p}\right) \\
& \qquad \text { for } t \in \bar{J}, p=0 \text { and } 2, r \geq p+2, \\
& \left\|\tilde{F}_{h} g(t)\right\| \leq C h^{3}\left\{\sup _{s \leq t}\|g(s)\|_{1}+t^{-1 / 2} \sup _{s \leq t}\left(\|g(s)\|+s\left\|g^{\prime}(s)\right\|\right)\right\} \\
& \quad \text { for } t \in J, r \geq 3
\end{aligned}
$$

and

$$
\left\|\tilde{F}_{h} g(t)\right\|_{1} \leq C h \sup _{s \leq t}\left(\|g(s)\|+s\left\|g^{\prime}(s)\right\|\right) \quad \text { for } t \in \bar{J}, r \geq 2 .
$$

Proof. In the same way as in (3.6) we obtain

$$
\begin{aligned}
t \tilde{F}_{h} g(t) & =\int_{0}^{t}(t-s) F_{h}(t-s) g(s) d s+\int_{0}^{t} s F_{h}(t-s) g(s) d s \\
& =t H_{h}(0) g(t)+\int_{0}^{t}\left\{\left((t-s) F_{h}(t-s)-H_{h}(t-s)\right) g(s)\right. \\
& \left.-s H_{h}(t-s) g^{\prime}(s)\right\} d s .
\end{aligned}
$$

The estimate (4.7) now follows by straightforward application of the estimates (4.3), (4.4), (1.7) and (1.8) for $H_{h}(t)$ and $F_{h}(t)$ (note that $H_{h}(0)=T_{h}-T$ ).

Similarly, we have for $r \geq 3$

$$
\begin{aligned}
\left\|\tilde{F}_{h} g(t)\right\| & \leq C h^{3}\left\{\sup _{s \leq t}\|g(s)\|_{1}+C t^{-1} \int_{0}^{t}(t-s)^{-1 / 2}\left(\|g(s)\|+s\left\|g^{\prime}(s)\right\|\right) d s\right\} \\
& \leq C h^{3}\left\{\sup _{s \leq t}\|g(s)\|_{1}+t^{-1 / 2} \sup _{s \leq t}\left(\|g(s)\|+s\left\|g^{\prime}(s)\right\|\right)\right\}
\end{aligned}
$$

proving (4.8), and (4.9) follows in the same way if we also utilize the fact

$$
\left\|F_{h}(t) v\right\|_{1} \leq C h t^{-1}\|v\| \quad \text { for } v \in L_{2}, r \geq 2 .
$$

To prove this last estimate, we note that $\theta_{0}(t)=E_{h}(t) P_{h} v-R_{h} E(t) v$ satisfies

$$
\left(e_{0, t}, \chi\right)+\left(\nabla \theta_{0}, \nabla \chi\right)=0 \text { for } \chi \in S_{h},
$$

and hence by known estimates,

$$
\left\|\nabla \theta_{0}(t)\right\|^{2} \leq\left\|e_{0, t}(t)\right\|\left\|\theta_{0}(t)\right\| \leq C\left(t^{-1}\|v\|\right)\left(C h^{2} t^{-1}\|v\|\right) \leq C h^{2} t^{-2}\|v\|^{2} .
$$

Since $\nabla \rho_{0}$ may also be bounded by the right-hand side of $(4.10)$, this establishes the desired result.

We are now ready for the estimate needed for the term $e_{1}$ in (4.1). 
LEMMA 4.3. Under the assumption of Theorem 4.1 we have for $e_{1}(t)=$ $\tilde{F}_{h}(\tilde{B} u)(t)$

and

$$
\left\|e_{1}(t)\right\| \leq C h^{4-\beta}\|v\| \quad \text { for } t \in \bar{J}, \beta \leq 2
$$

$$
\left\|e_{1}(t)\right\|_{1} \leq C h\|v\| \quad \text { for } t \in \bar{J}, \beta=2 .
$$

Proof. When $\beta=0$ and $\beta=2$, we have by Theorem 3.1 and Lemma 3.5

$$
\begin{aligned}
|\tilde{B} u(s)|_{2-\beta}+s\left|(\tilde{B} u)^{\prime}(s)\right|_{2-\beta} & \leq C\|v\|+s|B(s, s) u(s)|_{2-\beta}+s\left|\tilde{B}_{t} u(s)\right|_{2-\beta} \\
& \leq C\|v\|+C s|u(s)|_{2}+s\left|\tilde{B}_{t} u(s)\right|_{2-\beta} \leq C\|v\| .
\end{aligned}
$$

The result therefore follows in these cases by (4.7) with $p=2-\beta$. For $\beta=1$ the estimate follows similarly by (4.8), Theorem 3.1 and Lemma 3.5 .

The last inequality of the lemma is a consequence of (4.9), Theorem 3.1 and Lemma 3.5.

We now turn to the term $e_{2}$ defined in (4.1). Since $e_{2} \in S_{h}$, we shall only need to bound $\left(e_{2}, \chi\right)$ for $\chi \in S_{h}$. We note that by our definitions

$$
\begin{aligned}
\left(E_{h}(t-s)\left(\tilde{B}_{h} u_{h}(s)-P_{h} \tilde{B} u(s)\right), \chi\right) & =\left(\tilde{B}_{h} u_{h}(s)-P_{h} \tilde{B} u(s), E_{h}(t-s) \chi\right) \\
& =\int_{0}^{s} B\left(s, \tau ; e(\tau), E_{h}(t-s) \chi\right) d \tau
\end{aligned}
$$

and hence, since $e(t)=F_{h}(t) v+\hat{e}(t)$,

$$
\begin{aligned}
\left(e_{2}(t), \chi\right)= & \int_{0}^{t} \int_{0}^{s} B\left(s, \tau ; F_{h}(\tau) v, E_{h}(t-s) \chi\right) d \tau d s \\
& +\int_{0}^{t} \int_{0}^{s} B\left(s, \tau ; \hat{e}(\tau), E_{h}(t-s) \chi\right) d \tau d s \\
= & e_{21}(t ; \chi)+e_{22}(t ; \chi) .
\end{aligned}
$$

For the purpose of estimating $e_{21}(t ; \chi)$ we define the functional

$$
\tilde{\tilde{B}}(t ; f, g)=\int_{0}^{t} \int_{0}^{s} B(s, \tau ; f(\tau), g(t-s)) d \tau d s
$$

and show the following lemma, where $\tilde{f}(t)$ denotes $\int_{0}^{t} f(s) d s$, and similarly for $\tilde{g}(t)$.

LEMMA 4.4. Under the appropriate regularity assumptions for $f$ and $g$, we have

$$
\begin{array}{r}
|\tilde{\tilde{B}}(t ; f, g)| \leq C \sup _{s \leq t}\left(|\tilde{f}(s)|_{\beta-\kappa}+s|f(s)|_{\beta-\kappa}\right) \sup _{s \leq t}\left(|\tilde{g}(s)|_{\kappa}+s|g(s)|_{\kappa}\right) \\
\text { for } t \in \bar{J}, 0 \leq \kappa \leq 2, \beta=0 \text { and } 2
\end{array}
$$

and

$$
|\tilde{\tilde{B}}(t ; f, g)| \leq C \sup _{s \leq t}\left(s^{1 / 2}\|\tilde{f}(s)\|\right) \sup _{s \leq t}\left(s^{1 / 2}|g(s)|_{1}\right) \quad \text { for } t \in \bar{J}, \beta=1 .
$$

Proof. By integration by parts we get

$$
\begin{aligned}
\tilde{\tilde{B}}(t ; f, g)= & \int_{0}^{t} B(s, s ; \tilde{f}(s), g(t-s)) d s \\
& -\int_{0}^{t} \int_{0}^{s} B_{\tau}(s, \tau ; \tilde{f}(\tau), g(t-s)) d \tau d s \\
= & \sum_{j=1}^{2} \tilde{\tilde{B}}_{j}(t ; f, g) .
\end{aligned}
$$


Multiplying by $t$ and using integration by parts once more, we obtain for the first term

$$
\begin{aligned}
t \tilde{\tilde{B}}_{1}(t ; f, g)= & \int_{0}^{t} s B(s, s ; \tilde{f}(s), g(t-s)) d s \\
& +\int_{0}^{t}(t-s) B(s, s ; \tilde{f}(s), g(t-s)) d s \\
= & \int_{0}^{t} B(s, s ; \tilde{f}(s), \tilde{g}(t-s)) d s \\
& +\int_{0}^{t} s B_{s}(s, s ; \tilde{f}(s), \tilde{g}(t-s)) d s \\
& +\int_{0}^{t} s B(s, s ; f(s), \tilde{g}(t-s)) d s \\
& +\int_{0}^{t}(t-s) B(s, s ; \tilde{f}(s), g(t-s)) d s
\end{aligned}
$$

where $B_{s}$ is obtained by differentiating with respect to the first two arguments. In the second term in (4.14) we interchange the order of integration and integrate by parts again to obtain

$$
\begin{aligned}
\tilde{\tilde{B}}_{2}(t ; f, g)= & -\int_{0}^{t} \int_{\tau}^{t} B_{\tau}(s, \tau ; \tilde{f}(\tau), g(t-s)) d s d \tau \\
= & -\int_{0}^{t} B_{\tau}(\tau, \tau ; \tilde{f}(\tau), \tilde{g}(t-\tau)) d \tau \\
& -\int_{0}^{t} \int_{\tau}^{t} B_{s \tau}(s, \tau ; \tilde{f}(\tau), \tilde{g}(t-s)) d s d \tau .
\end{aligned}
$$

Together, (4.15) and (4.16) thus show

$$
\begin{aligned}
t \tilde{\tilde{B}}(t ; f, g)= & \int_{0}^{t} B(s, s ; \tilde{f}(s), \tilde{g}(t-s)) d s+\int_{0}^{t} s B_{s}(s, s ; \tilde{f}(s), \tilde{g}(t-s)) d s \\
& +\int_{0}^{t} s B(s, s ; f(s), \tilde{g}(t-s)) d s \\
& +\int_{0}^{t}(t-s) B(s, s ; \tilde{f}(s), g(t-s)) d s \\
& -t \int_{0}^{t} B_{s}(s, s ; \tilde{f}(s), \tilde{g}(t-s)) d s \\
& -t \int_{0}^{t} \int_{\tau}^{t} B_{s \tau}(s, \tau ; \tilde{f}(\tau), \tilde{g}(t-s)) d s d \tau
\end{aligned}
$$

By considering various possibilities for $\beta=0$ and $2,0 \leq \kappa \leq 2$, we find

$$
|B(\cdot, \cdot ; f, g)| \leq C|f|_{\beta-\kappa}|g|_{\kappa} \quad \text { for } \beta=0 \text { and } 2,0 \leq \kappa \leq 2,
$$

and similarly for $B_{s}$ and $B_{s \tau}$. Using this in (4.17) yields at once the first result of Lemma 4.4.

The second result of the lemma follows directly from (4.14), since

$$
|B(\cdot, \cdot ; f, g)| \leq C\|f\||g|_{1} \quad \text { for } \beta=1,
$$


and similarly for $B_{\tau}$. The reason why we are treating $\beta=1$ separately is that for $\beta=1, \kappa=2$ the factor $|f|_{-1}$ in (4.18) would have to be replaced by $\|f\|_{-1}$, since $\nabla g$ does not generally vanish on $\partial \Omega$ and this last norm is undesirable in our application below.

We are now ready to estimate the term $e_{21}(t ; \chi)$ in $(4.11)$.

LEMMA 4.5. We have

$$
\left|e_{21}(t ; \chi)\right| \leq C h^{4-\beta}\|v\|\|\chi\| \quad \text { for } t \in \bar{J}, \chi \in S_{h}, \beta \leq 2,
$$

and

$$
\left|e_{21}(t ; \chi)\right| \leq C h\|v\||\chi|_{-1, h} \quad \text { for } t \in \bar{J}, \chi \in S_{h}, \beta=2 .
$$

Proof. We first discuss $\beta=0$ and 2 . We have

$$
\begin{aligned}
e_{21}(t ; \chi) & =\tilde{\tilde{B}}\left(t ; F_{h} v, E_{h} \chi\right)=\tilde{\tilde{B}}\left(t ; F_{h} v, F_{h} \chi\right)+\tilde{\tilde{B}}\left(t ; F_{h} v, E \chi\right) \\
& =e_{211}(t ; \chi)+e_{212}(t ; \chi) .
\end{aligned}
$$

We shall estimate the two terms in the right-hand side individually.

With $\sim$ as usual denoting integrals over $\bar{J}$, we find, since $\left(F_{h} v\right) \sim(t)=-H_{h}(t) v+$ $H_{h}(0) v$, that by (4.12), (1.7), (4.3) and (4.10), with $\kappa=\min (\beta, 1)$,

$$
\begin{aligned}
& \left|e_{211}(t ; \chi)\right| \\
& \quad \leq C \sup _{s \leq t}\left(\left|H_{h}(s) v\right|_{\beta-\kappa}+s\left|F_{h}(s) v\right|_{\beta-\kappa}\right) \sup _{s \leq t}\left(\left|H_{h}(s) \chi\right|_{\kappa}+s\left|F_{h}(s) \chi\right|_{\kappa}\right) \\
& \quad \leq C h^{2-(\beta-\kappa)} h^{2-\kappa}\|v\|\|\chi\| \leq C h^{4-\beta}\|v\|\|\chi\| .
\end{aligned}
$$

Since $(E v)^{\sim}(t)=-T E(t) v+T v$, we obtain similarly

$$
\begin{aligned}
& \left|e_{212}(t ; \chi)\right| \\
& \quad \leq C \sup _{s \leq t}\left(\left|H_{h}(s) v\right|_{\beta-2}+s\left|F_{h}(s) v\right|_{\beta-2}\right) \sup _{s \leq t}\left(|T E(s) \chi|_{2}+s|E(s) \chi|_{2}\right) \\
& \quad \leq C h^{4-\beta}\|v\|\|\chi\| .
\end{aligned}
$$

Together, (4.19), (4.20) and (4.21) imply the first result of the lemma.

When $\beta=2$, we also have, since $\left(E_{h} v\right)^{\sim}(t)=-T_{h} E_{h}(t) v+T_{h} v$,

$$
\begin{aligned}
\left|e_{21}(t ; \chi)\right| & \leq C \sup _{s \leq t}\left(\left|H_{h}(s) v\right|_{1}+s\left|F_{h}(s) v\right|_{1}\right) \sup _{s \leq t}\left(\left|T_{h} E_{h}(s) \chi\right|_{1}+s\left|E_{h}(s) \chi\right|_{1}\right) \\
& \leq C h\|v\||\chi|_{-1, h},
\end{aligned}
$$

where in the last step we have used the following inequality (cf. [6, Lemma 3]) for the case $p=q=1$ :

$$
\left|E_{h}(t) \chi\right|_{q, h} \leq C t^{-(p-q) / 2}|\chi|_{p, h} \text { for } t \in J, p \leq q .
$$

When $\beta=1$, we have by Lemma 4.1, (4.13) and (4.22)

$$
\left|e_{21}(t ; \chi)\right| \leq C \sup _{s \leq t}\left(s^{1 / 2}\left\|H_{h}(s) v\right\|\right) \sup _{s \leq t}\left(s^{1 / 2}\left|E_{h}(s) \chi\right|_{1}\right) \leq C h^{3}\|v\|\|\chi\| .
$$

The proof is now complete.

Proof of Theorem 4.1. We shall now complete the proof of Theorem 4.1 by showing the estimate (4.2) for $\hat{e}=e_{1}+e_{2}$ and consider first the case $\beta \leq 1$. Recalling the notation of (4.11), we have now by (4.22),

$$
\left|e_{22}(t ; \chi)\right| \leq C \int_{0}^{t} \int_{0}^{s}\|\hat{e}(\tau)\|\left\|E_{h}(t-s) \chi\right\|_{\beta} d \tau d s \leq C \int_{0}^{t}\|\hat{e}\| d \tau\|\chi\| .
$$


Hence, using also Lemmas 4.3 and 4.5 , we find

$$
\|\hat{e}(t)\| \leq\left\|e_{1}(t)\right\|+\left\|e_{2}(t)\right\| \leq C h^{4-\beta}\|v\|+C \int_{0}^{t}\|\hat{e}\| d s,
$$

from which the desired result follows by Gronwall's lemma.

We turn to $\beta=2$. Now

$$
\begin{aligned}
e_{22}(t ; \chi)= & \int_{0}^{t} \int_{0}^{s} B\left(s, \tau ; \hat{e}(\tau), F_{h}(t-s) \chi\right) d \tau d s \\
& +\int_{0}^{t} \int_{0}^{s}\left(\hat{e}(\tau), B(s, \tau)^{*} E(t-s) \chi\right) d \tau d s \\
= & e_{221}(t ; \chi)+e_{222}(t ; \chi) .
\end{aligned}
$$

Interchanging the order of integration and integrating by parts, we have

$$
\begin{aligned}
e_{221}(t ; \chi)= & \int_{0}^{t} \int_{\tau}^{t} B\left(s, \tau ; \hat{e}(\tau), F_{h}(t-s) \chi\right) d s d \tau \\
= & \int_{0}^{t} B\left(t, \tau ; \hat{e}(\tau), H_{h}(0) \chi\right) d \tau-\int_{0}^{t} B\left(\tau, \tau ; \hat{e}(\tau), H_{h}(t-\tau) \chi\right) d \tau \\
& -\int_{0}^{t} \int_{\tau}^{t} B_{s}\left(s, \tau ; \hat{e}(\tau), H_{h}(t-s) \chi\right) d s d \tau
\end{aligned}
$$

Estimating these three terms individually gives, using (4.3) with $p=1, q=-1$,

$$
\left|e_{221}(t ; \chi)\right| \leq C \int_{0}^{t}\|\hat{e}\|_{1} d s \sup _{s \leq t}\left\|H_{h}(s) \chi\right\|_{1} \leq C h \int_{0}^{t}\|\hat{e}\|_{1} d s\|\chi\| .
$$

Similarly, we get

$$
\left|e_{222}(t ; \chi)\right| \leq C \int_{0}^{t}\|\hat{e}\| d s \sup _{s \leq t}\|T E(s) \chi\|_{2} \leq C \int_{0}^{t}\|\hat{e}\| d s\|\chi\| .
$$

Thus,

$$
\left|e_{22}(t ; \chi)\right| \leq C h \int_{0}^{t}\|\hat{e}\|_{1} d s\|\chi\|+C \int_{0}^{t}\|\hat{e}\| d s\|\chi\|,
$$

which together with Lemma 4.5 yields

$$
\left\|e_{2}(t)\right\| \leq C h^{2}\|v\|+C h \int_{0}^{t}\|\hat{e}\|_{1} d s+C \int_{0}^{t}\|\hat{e}\| d s .
$$

We shall prove presently that, when $\beta=2$, we have

$$
\|\hat{e}(s)\|_{1} \leq C h\|v\| \text { for } s \in \bar{J} \text {. }
$$

Assuming this for a moment, and using also Lemma 4.3, we obtain that

$$
\|\hat{e}(t)\| \leq\left\|e_{1}(t)\right\|+\left\|e_{2}(t)\right\| \leq C h^{2}\|v\|+C \int_{0}^{t}\|\hat{e}\| d s
$$

which concludes the proof of (4.2) as above.

It now remains only to prove (4.23). This time we write

$$
\begin{aligned}
e_{22}(t ; \chi)= & \int_{0}^{t} \int_{\tau}^{t} B\left(s, \tau ; \hat{e}(\tau), E_{h}(t-s) \chi\right) d s d \tau \\
= & \int_{0}^{t} B\left(t, \tau ; \hat{e}(\tau), T_{h} E_{h}(0) \chi\right) d s d \tau-\int_{0}^{t} B\left(\tau, \tau ; \hat{e}(\tau), T_{h} E_{h}(t-\tau) \chi\right) d \tau \\
& -\int_{0}^{t} \int_{\tau}^{t} B_{s}\left(s, \tau ; \hat{e}(\tau), T_{h} E_{h}(t-s) \chi\right) d s d \tau
\end{aligned}
$$


whence by (4.22)

$$
\left|e_{22}(\chi)\right| \leq C \int_{0}^{t}\|\hat{e}\|_{1} d s \sup _{s \leq t}\left\|T_{h} E_{h}(s) \chi\right\|_{1} \leq C \int_{0}^{t}\|\hat{e}\|_{1} d s\|\chi\|_{-1, h} .
$$

Applying the second estimate of Lemma 4.5, we have

$$
\left|\left(e_{2}(t), \chi\right)\right| \leq C\left\{h\|v\|+\int_{0}^{t}\|\hat{e}\|_{1} d s\right\}\|\chi\|_{-1, h},
$$

and hence, by duality,

$$
\left\|e_{2}(t)\right\|_{1} \leq C\left\{h\|v\|+\int_{0}^{t}\|\hat{e}\|_{1} d s\right\} .
$$

Together with the second estimate of Lemma 4.3, this shows

$$
\|\hat{e}(t)\|_{1} \leq\left\|e_{1}(t)\right\|_{1}+\left\|e_{2}(t)\right\|_{1} \leq C h\|v\|+C \int_{0}^{t}\|\hat{e}\|_{1} d s .
$$

By Gronwall's lemma, the proof of (4.23), and hence of the theorem, is now complete.

Remark 4.1. Theorem 4.1 remains valid when the approximation (1.2) is weakened to (2.13). In fact, for $\beta \leq 1$, only the $L_{2}$ norm error estimate for $R_{h}$ is needed in the proof, and for $\beta=2$ it suffices to consider $r=2$, in which case (2.13) implies (1.2).

Department of Mathematics

Chalmers University of Technology

S-412 96 Göteborg, Sweden

E-mail: cmsvt@seguc21.bitnet

naiying@math.chalmers.se

1. J. H. Bramble, A. H. Schatz, V. Thomée \& L. B. Wahlbin, "Some convergence estimates for semidiscrete Galerkin type approximations for parabolic equations," SIAM J. Numer. Anal., v. 14, 1977, pp. 218-241.

2. J. R. CANNON \& Y. LIN, "A priori $L^{2}$ error estimates for Galerkin methods for nonlinear parabolic integro-differential equations," Manuscript, 1987.

3. E. GREenWELl YANIK \& G. FAIRWEATHER, "Finite element methods for parabolic and hyperbolic partial integro-differential equations," Nonlinear Analysis Theory, Methods \& Applications, v. 12, 1988, pp. 785-809.

4. M.-N. LE ROUX \& V. THOMÉE, "Numerical solution of semilinear integro-differential equations of parabolic type with nonsmooth data," SIAM J. Numer. Anal. (To appear.)

5. I. H. SLOAN \& V. THOMÉE, "Time discretization of an integro-differential equation of parabolic type," SIAM J. Numer. Anal., v. 23, 1986, pp. 1052-1061.

6. V. THOMÉE, "Negative norm estimates and superconvergence in Galerkin methods for parabolic problems," Math. Comp., v. 34, 1980, pp. 93-113.

7. V. ThOMÉE, Galerkin Finite Element Methods for Parabolic Problems, Lecture Notes in Math., vol. 1054, Springer-Verlag, 1984.

8. M. F. WheELER, "A priori $L_{2}$ error estimates for Galerkin approximations to parabolic partial differential equations," SIAM J. Numer. Anal., v. 10, 1973, pp. 723-759. 